\title{
Multiparametric Magnetic Resonance Imaging
}

National Cancer Institute

\section{Source}

National Cancer Institute. Multiparametric Magnetic Resonance Imaging. NCI

Thesaurus. Code C116458.

The combination of multiple magnetic resonance techniques, including diffusion weighted imaging, dynamic contrast-enhanced imaging, and spectroscopy, to achieve an image that will allow for better identification of tumor size and location, as well as possibly identifying cancer spread and aggressiveness. 